\title{
Outcomes after hepatic encephalopathy in population-based cohorts of patients with cirrhosis
}

\author{
Elliot B. Tapper ${ }^{1,2}$ (D) | Devin Aberasturi ${ }^{3}$ | Zhe Zhao ${ }^{3}$ | Chia-Yang Hsu ${ }^{1}$ | \\ Neehar D. Parikh ${ }^{1}$
}

${ }^{1}$ Division of Gastroenterology and Hepatology, University of Michigan, Ann Arbor, MI, USA

${ }^{2}$ Gastroenterology Section, VA Ann Arbor Healthcare System, Ann Arbor, MI, USA

${ }^{3}$ Department of Biostatistics, University of Michigan, Ann Arbor, MI, USA

\section{Correspondence}

Elliot B. Tapper, MD 3912 Taubman, SPC 5362, 1500 E Medical Center Dr, Ann Arbor, MI 48109, USA.

Email: etapper@umich.edu

\section{Funding information}

Elliot Tapper receives funding from the National Institutes of Health through the Michigan Institute for Clinical and Health Research (KL2TR002241) and NIDDK (1K23DK117055-01A1). This work was supported in part by an unrestricted research grant from Valeant Pharmaceuticals (makers of Rifaximin). Valeant had no access to the data at any point and played no role in the planning of the study or the analysis or interpretation of the data.

\section{Summary}

Background: Hepatic encephalopathy is a devastating complication of cirrhosis.

Aim: To describe the outcomes after developing hepatic encephalopathy among contemporary, aging patients.

Methods: We examined data for a $20 \%$ random sample of United States Medicare enrolees with cirrhosis and Part D prescription coverage from 2008 to 2014. Among 49164 persons with hepatic encephalopathy, we evaluated the associations with transplant-free survival using Cox proportional hazard models with time-varying covariates (hazard ratios, HR) and incidence rate ratios (IRR) for healthcare utilisation measured in hospital-days and 30-day readmissions per person-year. We validated our findings in an external cohort of 2184 privately insured patients with complete laboratory values.

Results: Hepatic encephalopathy was associated with median survivals of 0.95 and 2.5 years for those $\geq 65$ or $<65$ years old and 1.1 versus 3.9 years for those with and without ascites. Non-alcoholic fatty-liver disease posed the highest adjusted risk of death among aetiologies, HR $1.0795 \% \mathrm{Cl}(1.02,1.12)$. Both gastroenterology consultation and rifaximin utilisation were associated with lower mortality, respective adjusted-HR $0.7395 \% \mathrm{Cl}(0.67,0.80)$ and $0.4095 \% \mathrm{Cl}(0.39,0.42)$. Thirty-day readmissions were fewer for patients seen by gastroenterologists $(0.7195 \% \mathrm{Cl}$ [0.57$0.88])$ and taking rifaximin (0.18 95\% $\mathrm{Cl}$ [0.08-0.40]). Lactulose alone was associated with fewer hospital-days, IRR $0.3195 \% \mathrm{Cl}(0.30-0.32)$, than rifaximin alone, $0.4995 \%$ $\mathrm{Cl}$ (0.45-0.53), but the optimal therapy combination was lactulose/rifaximin, IRR 0.28 $95 \% \mathrm{Cl}(0.27-0.30)$. These findings were validated in the privately insured cohort adjusting for model for endstage liver disease-sodium score and serum albumin.

Conclusions: Hepatic encephalopathy remains morbid and associated with poor outcomes among contemporary patients. Gastroenterology consultation and combination lactulose-rifaximin are both associated with improved outcomes. These data inform the development of care coordination efforts for subjects with cirrhosis. 


\section{1 | INTRODUCTION}

Cirrhosis is increasingly common. Its prevalence has doubled in the last decade. ${ }^{1}$ Furthermore, mortality due to cirrhosis rose by $65 \%$ from 2008 to 2016 without sign of slowing. ${ }^{2-7}$ The complexity and costs of cirrhosis are linked most closely to the complications of cirrhosis. Among these complications, none carry a more abrupt increase in mortality than hepatic encephalopathy. ${ }^{8-13}$ Hepatic encephalopathy is the most potent risk factor for hospitalisation, accidental trauma and mortality. ${ }^{14-17}$ The estimated incidence of hepatic encephalopathy is 11.6 per 100 -person years, ${ }^{18}$ rises to $40 \%$ by 5 years and is accompanied by a survival $<40 \%$ by 12 months after the development of hepatic encephalopathy. ${ }^{11,13}$ However, the epidemiology of cirrhosis has shifted, ${ }^{7}$ with an unclear impact on patient outcomes after developing hepatic encephalopathy in a contemporary cohort.

Driven by emerging risk factors, such as non-alcoholic fatty liver disease (NAFLD), ${ }^{4}$ patients with cirrhosis are presenting at increasingly older ages. ${ }^{19,20}$ Data regarding the outcomes of hepatic encephalopathy have been drawn from younger ( $<60$ years old) cohorts of patients without access to contemporary supportive care. ${ }^{8-13}$ In recent years there is increasing awareness of the benefits of nutritional support, ${ }^{21,22}$ mounting data on the benefits of coordinated care with subspecialists ${ }^{23}$ and novel pharmacotherapies for secondary prophylaxis of hepatic encephalopathy episodes. ${ }^{21}$ Optimised therapy using combination lactulose and rifaximin may not only prevent episodes of hepatic encephalopathy but also could reduce overall mortality. ${ }^{24-26}$

Herein, we evaluate the clinical outcomes after hepatic encephalopathy in two population-based cohort of patients with cirrhosis, focusing on 49000 Medicare enrolees and validating our findings in 2000 privately insured patients.

\section{2 | METHODS}

\section{1 | Study Population}

First, we examined data from a $20 \%$ random sample of US Medicare enrolees with cirrhosis (ICD9 571.2, 571.5 and 571.6) and continuous Part D (prescription) coverage from 2008 to 2014 (Figure S1). Patients were included from the time of their first cirrhosis diagnosis within the study period and were followed thereafter. A summary of diagnostic codes used is provided in Table S1. We included all patients who met criteria for cirrhosis using a coding algorithm validated for Medicare data ( $\geq 2$ diagnostic codes for cirrhosis). ${ }^{27}$ This study is a continuation of a prior examination of the incidence and risk factors for hepatic encephalopathy in the Medicare population. ${ }^{18}$ Subjects were followed until death, transplant or the end of study (12/31/2014 because ICD-10 replaced ICD-9 in 2015). In order to evaluate the impact of medication usage, we limited our analyses to beneficiaries enrolled in Medicare Part D. We excluded all patients with less than 90 days of outpatient follow up before developing HE. Second, we validated our findings in an external cohort of privately insured patients with cirrhosis with available laboratory data. Complete details of the validation cohort are available in the Supporting information. This study was approved by the University of Michigan institutional review board.

\section{2 | Definition of hepatic encephalopathy}

Incident hepatic encephalopathy was defined based on ICD-9 code 572.2 or the prescription of lactulose or rifaximin for $>90$ days (less if death or transplantation occurred before 90 days), whichever came first. The diagnostic code (572.2) has a specificity of $95 \%-99 \%{ }^{28,29}$ As previously published, ${ }^{13,30}$ we maximised sensitivity for incident hepatic encephalopathy using pharmacy linkage to include prescription of medications that are specific for hepatic encephalopathy therapy.

\section{3 | Outcomes}

Our primary outcome was survival after hepatic encephalopathy. As below, we model this outcome in multiple ways including as transplant-free survival and accounting for the competing risks of transplant. Patients were followed until death, transplant or the end of study. Secondary outcomes included hospital-days per patient-year and 30 -day readmissions per patient-year.

\section{$2.4 \mid$ Covariates}

For complete description of the cohort and risk adjustment we also included age, gender, race, Charlson Comorbidity Index (modified to exclude liver disease), ${ }^{31}$ liver disease aetiology, complications of cirrhosis and evaluation by a gastroenterologist/hepatologist. Patients could have multiple causes of cirrhosis (eg hepatitis B and C, hepatitis $C$ and alcohol-related liver disease). As performed by multiple investigators, we classified a group of patients with likely NAFLD-related cirrhosis who had cirrhosis (ICD-9 571.5) but lacked any diagnostic codes for viral hepatitis, alcohol-related use disorder or alcohol-related organ injury or autoimmune liver disease. ${ }^{32,33}$ For lack of specific codes for NAFLD, we refer to this as non-alcohol, nonviral-related cirrhosis. Liver disease severity was assessed using a combination of codes for diagnosis (eg ascites, variceal bleeding) and procedures (eg paracentesis and transjugular intrahepatic portosystemic shunt placement). We categorised hepatitis $C$ therapy using Part D dispensing records (Table S1). For the external cohort where we had access to laboratory data, we also adjusted for MELD-Na (model for end-stage liver disease-sodium), serum albumin and platelet count at the time of hepatic encephalopathy diagnosis.

We analysed two potentially modifiable factors. First, given that it has been associated with decreased hospitalisation risk 
in randomised and observational studies, ${ }^{21,34}$ we explored impact of rifaximin use on our outcomes adjusted for the covariates described above. Second, we evaluated the association of our primary and secondary outcomes with gastroenterology consultation.

\section{5 | Data analyses}

We used four analytic strategies to evaluate our outcomes accounting for biases.

- First, to account for varying trajectories of disease we employed multivariate Cox proportional hazard models for transplant-free survival using time-varying covariates with first-order autoregressive modelling. This method accounts for the temporal relationship of covariates (ie decompensation events during follow-up, progressive development of comorbidities, duration of exposure and the temporal proximity of the exposure to the outcome). We performed a sensitivity analysis of these data for persons diagnosed with hepatic encephalopathy as inpatients in an attempt to isolate those whose first diagnosis was with overt hepatic encephalopathy.

- Second, to account for diagnoses that developed but were not coded or treated prior to the transition from private to Medicare insurance, we repeated our analyses for all persons with a minimum of 1 year of follow-up without hepatic encephalopathy (ie washout).

- Third, we addressed the risk of residual immortal time bias despite the use of time-dependent Cox modelling using a Landmark analysis, ${ }^{35}$ setting the diagnosis of hepatic encephalopathy as time zero.

- Fourth, we employed Fine-Gray modelling to account for the competing risk of liver transplantation. ${ }^{36}$

- Fifth, we analysed an external/validation cohort where we could fully adjust our risk estimates using laboratory data (MELD-Na, albumin and platelet count). We included only patients with 1 year of follow-up without hepatic encephalopathy (washout) and performed all outcome assessments using a competing risk (death vs transplant) landmark analysis (setting the diagnosis of hepatic encephalopathy as time zero). We adjusted all analyses using variables that were present at the time of hepatic encephalopathy diagnosis (or within 30 days).

Hospital-days and readmissions were evaluated in negative binomial models and presented as the incidence per person-years and incidence rate ratios (IRR) with 95\% confidence intervals (CI). As the number of 30-day readmissions was left skewed (clustered around zero), we used a zero-inflated negative binomial model. The difference in separation of survival curves was evaluated using the logrank test. In all cases, the $P$-values presented were two-tailed with a $<0.05$ threshold for significance. All analyses were performed using $\mathrm{R}$ and SAS (SAS Institute Inc.).
TA B LE 1 Baseline Characteristics of Study Cohort

\begin{tabular}{|c|c|}
\hline $\begin{array}{l}\text { Clinical characteristics at the } \\
\text { time of hepatic encephalopathy } \\
\text { diagnosis }\end{array}$ & $\begin{array}{l}\text { Patients with hepatic } \\
\text { encephalopathy }(N=49164)\end{array}$ \\
\hline Age, median (IQR) & $63(55-71)$ \\
\hline Gender, male, n (\%) & $27515(56)$ \\
\hline White race, $\mathrm{n}(\%)$ & $38076(78)$ \\
\hline Medicaid co-insurance, n (\%) & 15 293(31) \\
\hline Urban, n (\%) & 39827 (81) \\
\hline \multicolumn{2}{|l|}{ Charlson Index } \\
\hline 0 & $17259(35)$ \\
\hline 1 & $17714(36)$ \\
\hline 2 & $10462(21)$ \\
\hline$\geq 3$ & $3324(7)$ \\
\hline End-stage renal disease, $\mathrm{n}(\%)$ & $1828(4)$ \\
\hline Disabled, n (\%) & $22259(46)$ \\
\hline \multicolumn{2}{|l|}{ Cirrhosis aetiology, n (\%) } \\
\hline Alcohol & $24183(50)$ \\
\hline Hepatitis C & $18352(38)$ \\
\hline Non-alcohol, nonviral & $15048(31)$ \\
\hline Hepatitis B & $2589(5)$ \\
\hline Ascites, n (\%) & $20771(43)$ \\
\hline Paracentesis, n (\%) & $6474(13)$ \\
\hline Varices, n (\%) & $10297(21)$ \\
\hline $\begin{array}{l}\text { Transjugular intrahepatic } \\
\text { portosystemic shunt, n(\%) }\end{array}$ & $704(1)$ \\
\hline Hepatocellular Carcinoma, n (\%) & $3361(7)$ \\
\hline Gastroenterology consult, n (\%) & $24090(49)$ \\
\hline
\end{tabular}

Abbreviations: $\mathrm{HE}$, hepatic encephalopathy.

Many patients had both alcohol-related and hepatitis $\mathrm{C}$ cirrhosis.

\section{3 | RESULTS}

\section{1 | Demographics and clinical characteristics of the medicare cohort}

Of the 49164 (26.4\%) patients diagnosed with hepatic encephalopathy, 50\% had alcohol-related cirrhosis and 38\% had HCV (including some with alcohol-related cirrhosis). At the time of hepatic encephalopathy diagnosis, $43 \%$ had ascites (13\% required paracentesis), $21 \%$ had varices and $7 \%$ had hepatocellular carcinoma (HCC). Overall, 49\% had received gastroenterology consultation prior to hepatic encephalopathy diagnosis (rising to $67 \%$ by the end of follow-up). Overall, 1620 persons had transjugular intrahepatic portosystemic shunts, 704 of whom received it prior to diagnosis of hepatic encephalopathy. Once hepatic encephalopathy was diagnosed, 28445 (57.8\%) received lactulose and 9615 (19.6\%) received rifaximin for at least 90 days $(31.2 \%$ received $<90$ days of either therapy). Of those who received prescriptions, the number of fills per person-year was $3.95 \pm 6.89$ and $3.83 \pm 6.75$ for lactulose and rifaximin respectively. 


\begin{tabular}{|c|c|c|c|c|}
\hline \multirow[b]{2}{*}{ Baseline Variable } & \multirow{2}{*}{$\begin{array}{l}\text { Death } \\
\text { Adjusted HR } \\
(95 \% \mathrm{CI})\end{array}$} & \multirow[b]{2}{*}{$P$-value } & \multirow{2}{*}{$\begin{array}{l}\text { Hospital days } \\
\text { Adjusted IRR } \\
(95 \% \mathrm{CI})\end{array}$} & \multirow[b]{2}{*}{$P$-value } \\
\hline & & & & \\
\hline Age (per year) & $1.02(1.02,1.03)$ & $<0.001$ & $1.01(1.01,1.01)$ & $<0.001$ \\
\hline Male & $1.21(1.19,1.24)$ & $<0.001$ & $1.03(0.99,1.06)$ & 0.116 \\
\hline End-stage renal disease & $1.08(1.01,1.14)$ & 0.015 & $1.15(1.06,1.25)$ & $<0.001$ \\
\hline Urban & $1.01(0.98,1.04)$ & 0.707 & $1.04(1.00,1.09)$ & 0.063 \\
\hline \multicolumn{5}{|l|}{ Race (relative to White) } \\
\hline Black & $1.00(0.96,1.04)$ & 0.960 & $1.17(1.10,1.23)$ & $<0.001$ \\
\hline Other & $0.90(0.87,0.94)$ & $<0.001$ & $0.97(0.92,1.03)$ & 0.353 \\
\hline \multicolumn{5}{|l|}{ Cirrhosis aetiology } \\
\hline Alcohol & $0.82(0.79,0.85)$ & $<0.001$ & $1.01(0.94,1.09)$ & 0.692 \\
\hline Hepatitis C & $0.87(0.85,0.90)$ & $<0.001$ & $1.20(1.15,1.25)$ & $<0.001$ \\
\hline Hepatitis B & $1.19(0.88,1.61)$ & 0.980 & $0.79(0.75,0.83)$ & $<0.001$ \\
\hline $\begin{array}{l}\text { Non-alcohol, Nonviral } \\
\text { cirrhosis }\end{array}$ & $1.07(1.02,1.12)$ & 0.004 & $0.98(0.93,1.03)$ & 0.427 \\
\hline \multicolumn{5}{|l|}{ Time-varying covariates } \\
\hline Gastroenterology Consult & $0.73(0.67,0.80)$ & $<0.001$ & $1.07(1.00,1.14)$ & 0.056 \\
\hline Rifaximin & $0.40(0.39,0.42)$ & $<0.001$ & $0.35(0.33,0.37)$ & $<0.001$ \\
\hline Ascites & $4.20(4.08,4.32)$ & $<0.001$ & $1.86(1.79,1.93)$ & $<0.001$ \\
\hline Varices & $1.03(1.00,1.06)$ & 0.029 & $0.77(0.74,0.80)$ & $<0.001$ \\
\hline $\begin{array}{l}\text { Transjugular intrahepatic } \\
\text { portosystemic shunt }\end{array}$ & $1.15(1.08,1.23)$ & $<0.001$ & $1.14(1.05,1.24)$ & 0.002 \\
\hline Hepatocellular carcinoma & $2.27(2,19,2.34)$ & $<0.001$ & $0.95(0.91,1.00)$ & 0.057 \\
\hline \multicolumn{5}{|c|}{ Charlson Comorbidity Index ( $\mathrm{CCl}$; relative to $\mathrm{CCl} 0)$} \\
\hline $\mathrm{CCl}=1$ & $1.20(1.17,1.24)$ & $<0.001$ & $1.17(1.13,1.22)$ & $<0.001$ \\
\hline $\mathrm{CCl}=2$ & $1.26(1.22,1.30)$ & $<0.001$ & $1.28(1.23,1.34)$ & $<0.001$ \\
\hline $\mathrm{CCl}=\geq 3$ & $1.42(1.35,1.48)$ & $<0.001$ & $1.33(1.24,1.42)$ & $<0.001$ \\
\hline
\end{tabular}

TABLE 2 Adjusted outcomes after a diagnosis of hepatic encephalopathy in a cohort of medicare enrolees

All estimates are adjusted for the variables presented in the table.

Abbreviations: $H R$, hazard ratio, IRR, incidence rate ratio. Non-alcoholic, nonviral = patients with cirrhosis codes but without any codes for alcohol-related diseases or viral hepatitis or autoimmune hepatitis.

\subsection{Mortality after the diagnosis of hepatic encephalopathy}

Overall, 1-year survival was $48.3 \%$ (median survival 0.92 years), including 58\% (median 1.4 years) for persons with hepatic encephalopathy defined by medications alone and $44.0 \%$ (median 0.76 years) for those with 572.2 ICD-9 codes (Table 1). Multiple clinical factors were associated with survival after HE. Age was a major determinant (Figure S3). For persons with cirrhosis aged $\geq 65$ years, the median survival overall was 0.95 years compared to 2.5 years for those $<65$ years old at the time of hepatic encephalopathy $(P<0.001)$. The sequence of decompensation was also important. The median survival was 1.1 years for those with ascites at the time of incident hepatic encephalopathy compared to 3.9 years for those without ascites $(P<0.001)$. For the 704 patients who developed hepatic encephalopathy after tranjugular portosystemic shunt placement, median survival was 0.88 years; it was 1.57 years for the 916 patients with transjugular intrahepatic portosystemic after hepatic encephalopathy (including a median of 0.41 years between hepatic encephalopathy development and shunt placement). Only $2.0 \%$ of patients underwent liver transplantation.

In Table 2, we present a multivariable Cox model for survival. The risk of death after hepatic encephalopathy increased with age, male gender, non-alcohol, nonviral-related cirrhosis, comorbidities including end-stage renal disease and other cirrhosis complications. Gastroenterology/hepatology consultation and rifaximin utilisation were associated with a lower risk of death, with respective adjusted hazard ratios of $0.7395 \% \mathrm{Cl}(0.67,0.80)$ and $0.4095 \%$ $\mathrm{Cl}(0.39,0.42)$. Figure 1 details how gastroenterology consultation and rifaximin use are inversely associated with mortality across multiple analytic strategies: when hepatic encephalopathy is first diagnosed as an inpatient, when the cohort is restricted to persons with 1 year of follow-up prior to hepatic encephalopathy diagnosis, and in both the landmark and competing-risk analysis (which 


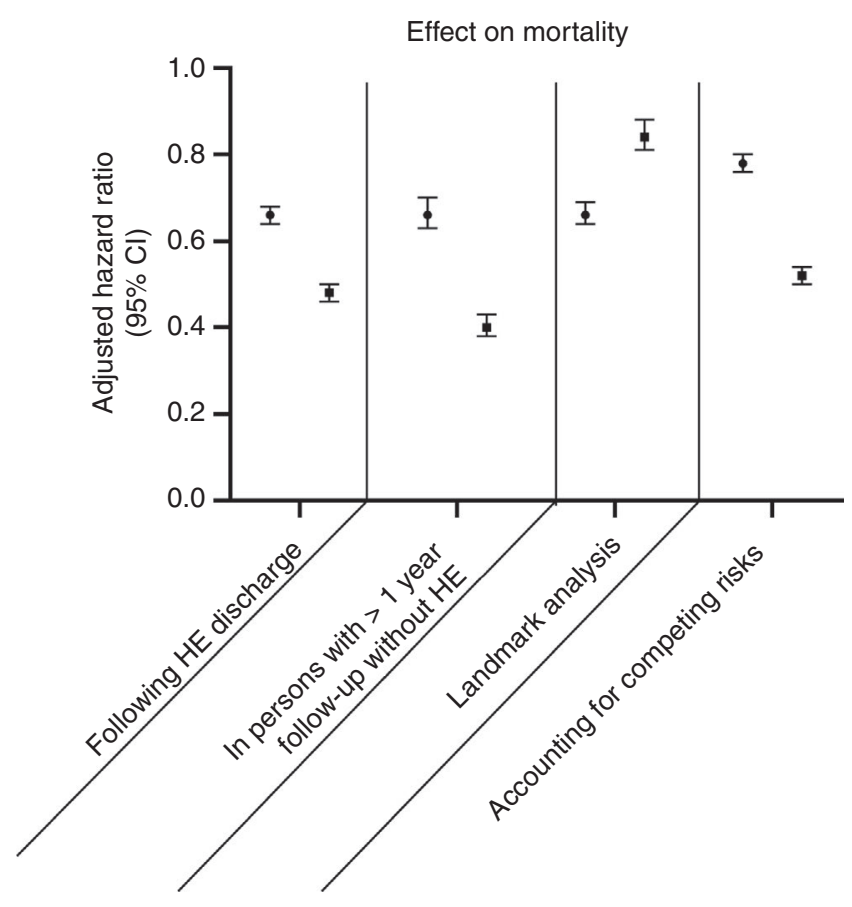

- Gastroenterology consult

- Rifaximin

FIGURE 1 The effect of gastroenterology consultation and rifaximin utilisation on mortality. The effect on mortality of gastroenterology consultation and rifaximin use are modelled for the subset of patients who were first diagnosed with hepatic encephalopathy as inpatients, in persons with $1 \mathrm{y}$ of 'washout' after enrolling in hepatic encephalopathy without recorded hepatic encephalopathy, as well as after a landmark analysis to extinguish the risk of immortal time bias and an analysis accounting for the competing risk of liver transplantation (the effect estimate in this case is a subhazard distribution ratio). $\mathrm{HE}$, hepatic encephalopathy

are further described with the effect estimates for all covariates in Tables S2-S4).

To further explore the effect of gastroenterology/hepatology consultation, we examined the impact of HCV therapy on hepatic encephalopathy outcomes. Overall, 2200 (12\%) of the patients with $\mathrm{HCV}$ underwent therapy, 1041 of whom received direct-acting antivirals. Among patients with HCV, median survival was 1.13, 2.10 and 2.12 years for patients receiving no therapy, direct-acting antiviral and interferon-based therapy.

\section{3 | Hospital utilisation after the diagnosis of hepatic encephalopathy}

Patients with hepatic encephalopathy were hospitalised for a median of 11.8 days (IQR2.9-38.0) per person-year. Table 2 provides the adjusted risk of hospitalisation by clinical covariate for patients after a diagnosis of hepatic encephalopathy. Notably, comorbid ascites was associated with the greatest burden of hospital utilisation, IRR $1.8695 \% \mathrm{Cl}$ (1.79-1.93). Overall, older age, number of comorbidities (including end-stage renal disease), hepatitis $\mathrm{C}$ and transjugular intrahepatic portosystemic shunt were all associated with more hospitaldays per person-year. Gastroenterology/hepatology consultation was not associated with a reduction adjusted risk for hospital utilisation but rifaximin use was, IRR $0.3595 \% \mathrm{Cl}(0.33,0.37)$.

In Figure 2, we show how rifaximin and gastroenterology consultation were associated hospital utilisation during multiple sensitivity analyses. First, we restricted the cohort to those whose hepatic encephalopathy was first diagnosed as inpatients; gastroenterology consultation with a modest increase in overall hospital-days while rifaximin co-therapy is still associated with fewer hospital-days when hepatic encephalopathy is first diagnosed as an inpatient, IRR $0.8095 \%$ $\mathrm{Cl}$ (0.76-0.84). Second, similar results were observed when the cohort was restricted to those with $\geq 1$-year follow-up prior to hepatic encephalopathy diagnosis. Third, we also evaluated the frequency of 30-day readmissions per person-year. Both gastroenterology consultation and rifaximin use were associated with lower all-cause 30-day readmissions, respective IRR $0.7195 \% \mathrm{Cl}(0.57-0.88)$ and $0.1895 \% \mathrm{Cl}(0.08-0.40)$. Finally, we examined the impact of various combinations of hepatic encephalopathy therapy on hospital-days. We set as the reference the many patients did not receive more than 90-days of hepatic encephalopathy therapy. Relative to 'no therapy', persons receiving lactulose alone had a markedly lower IRR for hospital-days per person-year than those receiving rifaximin alone, respective IRR $0.3195 \% \mathrm{Cl}(0.30-0.32)$ and $0.4995 \% \mathrm{Cl}(0.45-0.53)$. The relative IRR associated with combination lactulose and rifaximin was IRR $0.2895 \% \mathrm{Cl}(0.27-0.30)$.

\section{4 | External cohort of privately insured patients with hepatic encephalopathy}

In a cohort of 2184 patients with hepatic encephalopathy and an average age of 61, MELD-Na of 12 and albumin of 3.1, we found similar demographics, clinical factors and associations with mortality and hospital utilisation (Table 3). The cumulative incidence of death (accounting for competing risks) at 1,2 and 3 years was 0.19 (0.18-0.21), 0.29 (0.27-0.32) and 0.44 (0.40-0.48). As shown in Table S5, the risk of death was highest for patients $>65$ years old and those with pre-existing ascites. Gastroenterology consultation was much more common (85\% prior to hepatic encephalopathy and $90 \%$ during follow up) and was not statistically associated with mortality or utilisation, irrespective of whether it occurred during the index hospitalisation for hepatic encephalopathy. However, rifaximin use was associated with reduced mortality sHR 0.40 (0.39-0.42) and hospital-days IRR 0.35 (0.33-0.37).

\section{4 | DISCUSSION}

Hepatic encephalopathy is a watershed moment in the patient's experience of chronic liver disease, after which the risk of hospitalisation and death sharply rises. ${ }^{16,37,38}$ To evaluate the natural history and outcomes following the development of hepatic encephalopathy, we examined a large sample (>49 000 Medicare enrolees with 


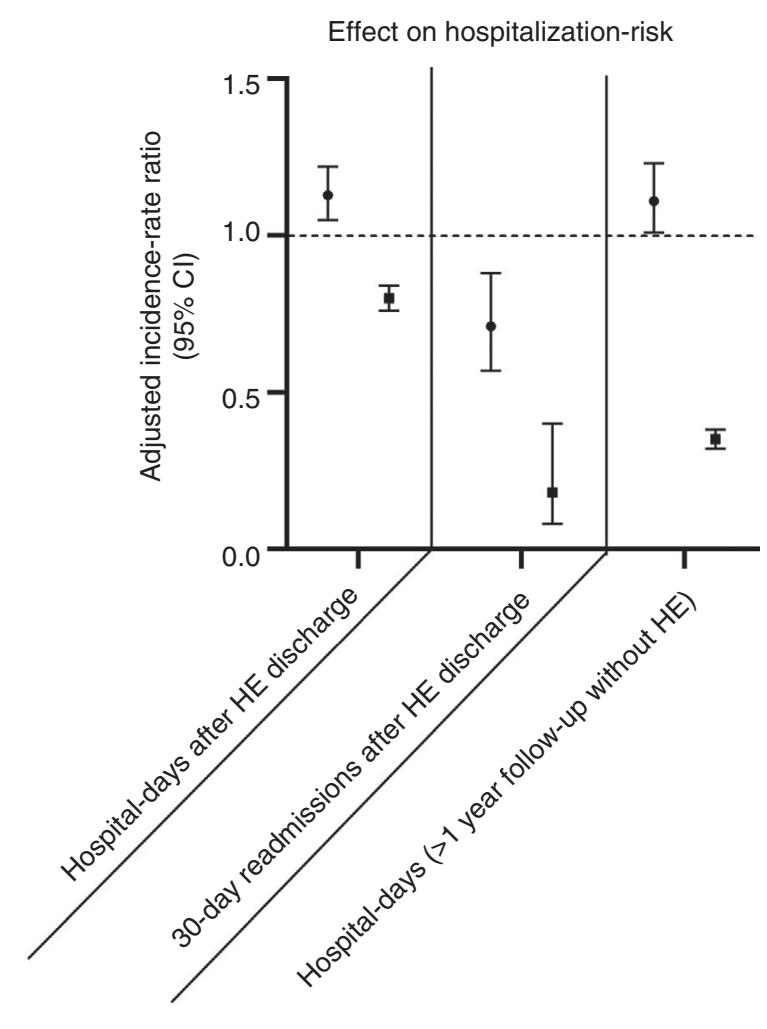

- Gastroenterology consult

- Rifaximin

FIGURE 2 The effect of gastroenterology consultation and rifaximin utilisation on healthcare utilisation after hepatic encephalopathy. The effect on hospitalisation associated with both gastroenterology consultation and rifaximin use. Gastroenterology consultation is associated with fewer 30-day readmissions but a higher number of hospital-days in the cohort after a hepatic encephalopathy discharge as well the cohort with 1 year of 'washout'. Rifaximin, by contrast, is associated with fewer hospitalisations in each analysis. $\mathrm{HE}$, hepatic encephalopathy

hepatic encephalopathy) with long-term follow-up and detailed patient-level characteristics for time-varying risk adjustment. We also validated our findings in a second cohort of $>2000$ privately-insured patients. These data extend our understanding of the natural history of hepatic encephalopathy in multiple ways.

\section{1 | Survival after hepatic encephalopathy for contemporary patients}

Survival is diminished following the development of hepatic encephalopathy with a median survival of 0.92 years. Furthermore, we found that while comorbidities and disease severity impact survival as expected. Non-alcohol, nonviral (mostly NAFLD) cirrhosis also increases the risk of death, adjusted HR $1.0795 \% \mathrm{Cl}(1.02,1.12)$. In the privately insured cohort where biochemical measures were available, only MELD-Na, albumin and HCC were associated with mortality. This study builds on prior estimates of survival after decompensation with hepatic encephalopathy, overt or covert, all drawn from the referral setting. For example, Bustamante et al reported $43 \%$ survival at 1-year in a cohort of younger persons (none with NAFLD), while Ampuero and Bajaj both demonstrated a higher risk of death or transplantation in persons with covert hepatic encephalopathy. ${ }^{15,39,40}$ In contrast, our study examines two population-based cohorts hundreds-fold larger with both variable exposure to subspecialist consultation and differences in the supportive care provided.

\subsection{Interventions associated with survival after hepatic encephalopathy}

Following the development of hepatic encephalopathy, few interventions beyond liver transplantation are associated with improved survival. For reasons thought to be related to improved quality of care, prior studies have found an association with improved survival after gastroenterology consultation for all-comers with chronic liver disease seen in the Veterans Affairs. ${ }^{41,42}$ However, fewer than $10 \%$ of the patients in these studies had cirrhosis. Furthermore, the impact of subspecialist involvement on patients with hepatic encephalopathy was not evaluated. ${ }^{41,42}$ We extend the association of gastroenterology in hepatic encephalopathy volvement with improved survival to a cohort of patients with hepatic encephalopathy with an adjusted hazard ratio of $0.73,95 \% \mathrm{Cl}(0.67-0.80)$. This association may be mediated by adherence to quality metrics (such as cancer or varices screening), ${ }^{23} \mathrm{HCV}$ therapy, ${ }^{43}$ referral for transplantation and use of guideline-concordant therapies for hepatic encephalopathy.

We also show that rifaximin use is associated with improved survival. Rifaxmin reduces the risk of hospitalisation for persons with hepatic encephalopathy. ${ }^{21,34}$ Hospitalisation itself is independently associated with mortality. ${ }^{44}$ Hospitalisation is associated with progressive debility and nosocomial complications making plausible the association between improved outcomes and interventions that safely avoid re-hospitalisation. Our study identifies a possible mortality benefit for rifaximin, adjusting for disease severity and gastroenterology consultation. This effect is also observed in our validation cohort where we could adjust for disease severity using laboratory data (ie MELD-Na). This effect is also robust to multiple analytic strategies to address biases due to competing risks (with transplant) and immortal time. Prior studies have suggested that rifaximin carries a mortality benefit. ${ }^{24-26}$ Most recently, Salehi et al showed that rifaximin was associated with decreased bleeding and infections in addition to reduced episodes in cohort of 101 transplant-waitlisted persons with hepatic encephalopathy. ${ }^{25}$ Our data extend these findings in a cohort hundreds-fold larger with variable exposure to subspecialty consultation while reinforcing that rifaximin monotherapy is inadequate.

\subsection{Hospital-days after hepatic encephalopathy}

Hepatic encephalopathy is the most potent risk factor for repeated hospitalisation for patients with cirrhosis. ${ }^{17}$ These data extend 
TABLE 3 Adjust outcomes after hepatic encephalopathy in a cohort of 2184 privately insured persons with cirrhosis

\begin{tabular}{|c|c|c|c|c|c|}
\hline \multirow[b]{2}{*}{ Baseline variable } & \multirow[b]{2}{*}{ Baseline value } & \multirow{2}{*}{$\begin{array}{l}\text { Death } \\
\text { Adjusted sHR (95\% } \\
\text { Cl) }\end{array}$} & \multirow[b]{2}{*}{$P$-value } & \multicolumn{2}{|l|}{ Hospital days } \\
\hline & & & & Adjusted IRR (95\%CI) & $P$-value \\
\hline Age (per year) & $61 \pm 14$ & $1.03(1.02-1.04)$ & $<0.001$ & $1(0.99-1.01)$ & 0.963 \\
\hline Male & $1334(57 \%)$ & $1.13(0.94-1.36)$ & 0.183 & $0.96(0.84-1.11)$ & 0.608 \\
\hline White Race (vs others) & $1460(62 \%)$ & $0.97(0.81-1.15)$ & 0.183 & $1.07(0.93-1.23)$ & 0.364 \\
\hline Alcohol & $1208(51 \%)$ & $0.85(0.71-1.01)$ & 0.069 & $0.9(0.78-1.04)$ & 0.139 \\
\hline Diabetes mellitus & $1264(54 \%)$ & $0.88(0.74-1.06)$ & 0.189 & $1.15(0.99-1.35)$ & 0.068 \\
\hline Hepatitis B & $199(8 \%)$ & $0.75(0.54-1.04)$ & 0.086 & $0.91(0.71-1.16)$ & 0.434 \\
\hline Hepatitis C & $1004(43 \%)$ & $0.86(0.71-1.04)$ & 0.114 & $0.91(0.79-1.06)$ & 0.231 \\
\hline Rifaximin & $492(21 \%)$ & $0.58(0.46-0.72)$ & $<0.001$ & $0.56(0.47-0.66)$ & $<0.001$ \\
\hline Ascites & 887 (38\%) & $1.15(0.95-1.39)$ & 0.165 & $1.13(0.98-1.3)$ & 0.091 \\
\hline Variceal bleeding & $169(7 \%)$ & $0.96(0.67-1.37)$ & 0.812 & $0.8(0.61-1.04)$ & 0.096 \\
\hline $\begin{array}{l}\text { Transjugular intrahepatic } \\
\text { portosystemic shunt }\end{array}$ & $59(3 \%)$ & $1.03(0.65-1.61)$ & 0.913 & $1.41(0.92-2.17)$ & 0.115 \\
\hline Hepatocellular carcinoma & 255 (11\%) & $1.7(1.31-2.20)$ & $<0.001$ & $1.49(1.2-1.86)$ & $<0.001$ \\
\hline \multicolumn{6}{|c|}{ Charlson comorbidity index $(\mathrm{CCl}$; relative to $\mathrm{CCl} 1)$} \\
\hline $\mathrm{CCl}=2$ & $356(15 \%)$ & $1.46(0.91-2.35)$ & 0.117 & $0.77(0.56-1.08)$ & 0.128 \\
\hline $\mathrm{CCl}=\geq 3$ & $1864(79 \%)$ & $1.1(0.8-1.50)$ & 0.06 & $0.94(0.66-1.34)$ & 0.736 \\
\hline
\end{tabular}

Abbreviations: MELD-Na (model for endstage liver disease-sodium), sHR, subhazard distribution ratio (accounts for the competing risk of death).

current knowledge both by highlighting the subgroups at highest risk and tools needed to curb hospitalisation risk. We show that older persons with alcohol-related liver disease, transjugular intrahepatic portosystemic shunts and multiple comorbidities are at higher risk of hospitalisation.

Receipt of appropriate hepatic encephalopathy therapy is crucial. Unfortunately, these data highlight gaps in medical therapy for persons with hepatic encephalopathy. As recently shown by Bajaj, many persons with hepatic encephalopathy are often not even prescribed lactulose after hospitalisation for hepatic encephalopathy. ${ }^{45}$ We too find that many do not receive lactulose. Lactulose therapy is associated with reduced hospital-days-IRR $0.3195 \% \mathrm{Cl}(0.30$ 0.32). Accordingly, efforts to optimise care begin with lactulose prescription. Conversely, rifaximin monotherapy is common. It appears to be substantially less effective than lactulose monotherapy at preventing hospitalisation. Conversely, adjusting for lactulose use, those who receive rifaximin co-therapy are at lower risk of hospitalisation-IRR $0.3595 \% \mathrm{Cl}(0.33,0.37)$. We also found that both gastroenterology consultation and rifaximin use were associated with lower all-cause 30-day readmissions per person-year, respective IRR $0.7195 \% \mathrm{Cl}(0.57-0.88)$ and $0.1895 \% \mathrm{Cl}(0.08-0.40)$. These data confirm the effects of rifaximin co-therapy on healthcare utilisation observed in prior randomised and prospective trials and extend these associations to the level of population-based data.22,35

\subsection{Optimal care for persons with hepatic encephalopathy}

In sum, these data consolidate findings from prior studies that speak to the nature of optimal care for patients at high risk of death and repeated hospitalisation. In a landmark trial, Morando et al showed that the risk of both death and readmission can be improved with a care co-ordination programme. ${ }^{46}$ Their multipronged intervention involved readily available gastroenterology consultation, testing for and treatment of cognitive dysfunction (ie with optimisation of lactulose and/or addition of rifaximin), testing for and treatment of alcohol-use disorder and on-demand procedures such as paracentesis. ${ }^{46}$ In contrast to this ideal, and echoing the findings of prior studies, ${ }^{41,47}$ we find that only half (49\%) of persons with cirrhosis are evaluated by a gastroenterologist prior to the development of hepatic encephalopathy. Access and referral to gastroenterologists 
is therefore a key target in the improvement of clinical outcomes and overall healthcare utilisation for persons with cirrhosis. Patients who see gastroenterologists are more likely to receive optimal therapy with lactulose and rifaximin, ${ }^{23}$ receive HCV therapy ${ }^{43}$ and timely referral for transplant evaluation. Finally, these data confirm the necessity of continuing lactulose after discharge for hepatic encephalopathy and suggest a role for rifaximin co-therapy for hepatic encephalopathy as part of best practice, particularly for those hospitalised with hepatic encephalopathy or at high risk for re-hospitalisation.

\subsection{Contextual factors}

Our findings must be interpreted in the context of the study design. First, laboratory results were not available to calculate model for endstage liver disease scores in the Medicare cohort. However, with laboratory data in the privately insured cohort, there was limited difference in the direction of effect. Second, we could not determine which patients with alcohol-related disease were actively drinking. Third, we have neither access to the staging of hepatic encephalopathy at diagnosis nor the results of any cognitive testing (if it was performed) and therefore it was possible that many patients had earlier stages of hepatic encephalopathy (ie covert hepatic encephalopathy). However, we noticed no difference outcomes for persons on the basis of inpatient versus outpatient diagnosis, suggesting that hepatic encephalopathy was a negative prognostic development irrespective of stage. Fourth, although we have prescription fill rates, we cannot speak to medication adherence. However, it is notable that even suboptimal fill rates, combination lactulose and rifaximin use was associated with improved outcomes. Fifth, although gastroenterology consultation would be expected to reflect sicker patients (confounding by indication), it was associated with lower risk of death and hospitalisation lending credence to a true effect. Subspecialty consultation access is complex and likely related to multiple unmeasured factors. Given the proportion with access in the private insurance cohort (90\%), we could not meaningfully validate this finding outside Medicare.

\section{5 | CONCLUSION}

Hepatic encephalopathy is common and morbid. These data provide the data necessary to inform contemporary patients of their prognosis and suggest a role for interventions that are linked to improved survival and reduced hospitalisation. Efforts to expand and co-ordinate access to expert consultation, reinforce lactulose use after discharge and reduce the cost barrier of rifaximin may be warranted to improve outcomes for the population with cirrhosis.

\section{ACKNOWLEDGEMENT}

Declaration of personal interests: Elliot Tapper has served as a consultant to Norvartis and Allergan, has served on advisory boards for Mallinckrodt and Bausch Health and has received unrestricted research grants from Gilead and Valeant. Valeant is the maker of Rifaximin, a medication approved for treatment of hepatic encephalopathy. Neehar Parikh has served as a consultant to Bristol MyersSquibb, Exelexis, Freenome, Eli Lilly, Fujifilm and Exact Sciences, has served on advisory boards for Merck, Exelexis, Bayer and Eisai and has received research funding from Bayer, Exact Sciences and Target Pharmasolutions. No other author has a conflict of interest.

\section{AUTHORSHIP}

Guarantor of this article: Elliot Tapper.

Author contributions: Tapper: Concept and Writing. Tapper, Aberasturi, Zhao, Hsu, Parikh: Analysis. Tapper, Aberasturi, Zhao, Hsu: Data acquisition. Aberasturi, Parikh, Zhao, Hsu: Revision

\section{ORCID}

Elliot B. Tapper (iD https://orcid.org/0000-0002-0839-1515

\section{REFERENCES}

1. Beste LA, Leipertz SL, Green PK, et al. Trends in burden of cirrhosis and hepatocellular carcinoma by underlying liver disease in US veterans, 2001-2013. Gastroenterology. 2015;149:1471-1482.e5.

2. Estes $\mathrm{C}$, Razavi H, Loomba R, et al. Modeling the epidemic of nonalcoholic fatty liver disease demonstrates an exponential increase in burden of disease. Hepatology. 2018;67:123-133

3. Moon A, Green P, Berry K, et al. Transformation of hepatitis C antiviral treatment in a national healthcare system following the introduction of direct antiviral agents. Aliment Pharmacol Ther. 2017;45:1201-1212.

4. Parikh ND, Marrero WJ, Wang J, et al. Projected increase in obesity and non-alcoholic steatohepatitis-related liver transplantation waitlist additions in the United States. Hepatology. 2019;70:487-495.

5. Younossi ZM, Stepanova M, Afendy M, et al. Changes in the prevalence of the most common causes of chronic liver diseases in the United States from 1988 to 2008. Clin Gastroenterol Hepatol. 2011;9:524-530.e1.

6. Tapper EB, Parikh ND. Mortality due to cirrhosis and liver cancer in the United States, 1999-2016: observational study. BMJ. 2018;362:k2817.

7. Moon AM, Singal AG, Tapper EB. Contemporary epidemiology of chronic liver disease and cirrhosis. Clin Gastroenterol Hepatol. 2019.https://doi.org/10.1016/j.cgh.2019.07.060 [Epub ahead of print].

8. Gomez EV, Rodriguez YS, Bertot LC, et al. The natural history of compensated HCV-related cirrhosis: a prospective long-term study. J Hepatol. 2013;58:434-444.

9. Dienstag JL, Ghany MG, Morgan TR, et al. A prospective study of the rate of progression in compensated, histologically advanced chronic hepatitis C. Hepatology. 2011;54:396-405.

10. Konerman MA, Zhang $\mathrm{Y}$, Zhu J, et al. Improvement of predictive models of risk of disease progression in chronic hepatitis $C$ by incorporating longitudinal data. Hepatology. 2015;61:1832-1841.

11. Ginés $P$, Quintero $E$, Arroyo $V$, et al. Compensated cirrhosis: natural history and prognostic factors. Hepatology. 1987;7:122-128.

12. Jepsen $P$, Ott $P$, Andersen $P K$, et al. Clinical course of alcoholic liver cirrhosis: A Danish population-based cohort study. Hepatology. 2010;51:1675-1682.

13. Tapper EB, Parikh N, Sengupta N, et al. A risk score to predict the development of hepatic encephalopathy in a population-based cohort of patients with cirrhosis. Hepatology. 2018;68:1498-1507. 
14. Bajaj JS, O'Leary JG, Tandon P, et al. Hepatic encephalopathy is associated with mortality in patients with cirrhosis independent of other extrahepatic organ failures. Clin Gastroenterol Hepatol. 2017; 15:565-574.e4.

15. Patidar KR, Thacker LR, Wade JB, et al. Covert hepatic encephalopathy is independently associated with poor survival and increased risk of hospitalization. Am J Gastroenterol. 2014;109:1757.

16. Ezaz G, Murphy SL, Mellinger J, et al. Increased morbidity and mortality associated with falls among patients with cirrhosis. Am J Med. 2018;131:645-650.

17. Tapper EB, Halbert B, Mellinger J. Rates of and reasons for hospital readmissions in patients with cirrhosis: a multistate population-based cohort study. Clin Gastroenterol Hepatol. 2016;14:1181-1188.

18. Tapper EB, Henderson JB, Parikh ND, et al. Incidence of and risk factors for hepatic encephalopathy in a population-based cohort of americans with cirrhosis. hepatology. Communications. 2019;3:1510-1519.

19. Scaglione S, Kliethermes S, Cao G, et al. The epidemiology of cirrhosis in the United States. J Clin Gastroenterol. 2015;49:690-696.

20. Sharpton SR, Feng S, Hameed B, et al. Combined effects of recipient age and model for end-stage liver disease score on liver transplantation outcomes. Transplantation. 2014;98:557.

21. Bass NM, Mullen KD, Sanyal A, et al. Rifaximin treatment in hepatic encephalopathy. N Engl J Med. 2010;362:1071-1081.

22. Bajaj JS, Acharya C, Fagan A, et al. Proton pump inhibitor initiation and withdrawal affects gut microbiota and readmission risk in cirrhosis. Am J Gastroenterol. 2018;113:1177-1186.

23. Tapper EB, Hao S, Lin M, et al. The quality and outcomes of care provided to patients with cirrhosis by advanced practice providers. Hepatology. 2020;71:225-234.

24. Sharma BC, Sharma P, Lunia MK, et al. A randomized, double-blind, controlled trial comparing rifaximin plus lactulose with lactulose alone in treatment of overt hepatic encephalopathy. Am J Gastroenterol. 2013;108:1458-1463.

25. Salehi S, Tranah TH, Lim S, et al. Rifaximin reduces the incidence of spontaneous bacterial peritonitis, variceal bleeding and all-cause admissions in patients on the liver transplant waiting list. Aliment Pharmacol Ther. 2019;50:435-441.

26. Kimer N, Krag A, Møller S, et al. Systematic review with meta-analysis: the effects of rifaximin in hepatic encephalopathy. Aliment Pharmacol Ther. 2014;40:123-132.

27. Rakoski MO, McCammon RJ, Piette JD, et al. Burden of cirrhosis on older Americans and their families: analysis of the health and retirement study. Hepatology. 2012;55:184-191.

28. Nehra MS, Ma Y, Clark C, et al. Use of administrative claims data for identifying patients with cirrhosis. J Clin Gastroenterol. 2013;47:e50.

29. Re VL, Lim JK, Goetz MB, et al. Validity of diagnostic codes and liver-related laboratory abnormalities to identify hepatic decompensation events in the Veterans Aging Cohort Study. Pharmacoepidemiol Drug Saf. 2011;20:689-699.

30. Tapper EB, Korovaichuk S, Baki J, et al. Identifying patients with hepatic encephalopathy using administrative data in the ICD-10 era. Clin Gastroenterol Hepatol. 2019. https://doi.org/10.1016/j. cgh.2019.12.017 [Epub ahead of print].

31. Deyo RA, Cherkin DC, Ciol MA. Adapting a clinical comorbidity index for use with ICD-9-CM administrative databases. J Clin Epidemiol. 1992;45:613-619.

32. Mellinger JL, Shedden K, Winder GS, et al. The High Burden Of Alcoholic Cirrhosis In Privately Insured Persons in the United States. Hepatology. 2018;68:872-882
33. Allen AM, Therneau TM, Larson JJ, et al. Nonalcoholic fatty liver disease incidence and impact on metabolic burden and death: A 20 year-community study. Hepatology. 2018;67:1726-1736.

34. Tapper EB, Finkelstein D, Mittleman MA, et al. A quality improvement initiative reduces 30-day rate of readmission for patients with cirrhosis. Clin Gastroenterol Hepatol. 2016;14:753-759.

35. Anderson JR, Cain KC, Gelber RD. Analysis of survival by tumor response. J Clin Oncol. 1983;1:710-719.

36. Fine JP, Gray RJ. A proportional hazards model for the subdistribution of a competing risk. J Am Stat Assoc. 1999;94:496-509.

37. Tapper EB, Risech-Neyman Y, Sengupta N. Psychoactive medications increase the risk of falls and fall-related injuries in hospitalized patients with cirrhosis. Clin Gastroenterol Hepatol. 2015;13:1670-1675.

38. Bajaj JS, Saeian K, Schubert CM, et al. Minimal hepatic encephalopathy is associated with motor vehicle crashes: the reality beyond the driving test. Hepatology. 2009;50:1175-1183.

39. Ampuero J, Simón M, Montoliú C, et al. Minimal hepatic encephalopathy and critical flicker frequency are associated with survival of patients with cirrhosis. Gastroenterology. 2015;149:1483-1489.

40. Bustamante J, Rimola A, Ventura P-J, et al. Prognostic significance of hepatic encephalopathy in patients with cirrhosis. J Hepatol. 1999;30:890-895.

41. Mellinger JL, Moser S, Welsh DE, et al. Access to subspecialty care and survival among patients with liver disease. Am J Gastroenterol. 2016;111:838.

42. Su GL, Glass L, Tapper EB, et al. Virtual consultations through the Veterans administration SCAN-ECHO project improves survival for Veterans with liver disease. Hepatology. 2018;68:2317-2324.

43. Tapper EB, Parikh ND, Green PK, et al. Reduced Incidence of hepatic encephalopathy and higher odds of resolution associated with eradication of HCV infection. Clin Gastroenterol Hepatol. 2020;18:1197-1206.

44. Volk ML, Tocco RS, Bazick J, et al. Hospital readmissions among patients with decompensated cirrhosis. Am J Gastroenterol. 2012;107:247.

45. Bajaj JS, O'Leary JG, Tandon P, et al. Targets to improve quality of care for patients with hepatic encephalopathy: data from a multi-centre cohort. Aliment Pharmacol Therap. 2019;49:1518-1527.

46. Morando F, Maresio G, Piano S, et al. How to improve care in outpatients with cirrhosis and ascites: a new model of care coordination by consultant hepatologists. J Hepatol. 2013;59:257-264.

47. Mellinger JL, Volk ML. Multidisciplinary management of patients with cirrhosis: a need for care coordination. Clin Gastroenterol Hepatol. 2013;11:217-223.

\section{SUPPORTING INFORMATION}

Additional supporting information will be found online in the Supporting Information section.

How to cite this article: Tapper EB, Aberasturi D, Zhao Z, Hsu C-Y, Parikh ND. Outcomes after hepatic encephalopathy in population-based cohorts of patients with cirrhosis. Aliment Pharmacol Ther. 2020;51:1397-1405. https://doi.org/10.1111/ apt.15749 